\title{
Synthetic and natural antioxidants attenuate cisplatin-induced vomiting
}

\author{
Javaid Alam ${ }^{1}$, Fazal Subhan ${ }^{1 *}$ D, Ihsan Ullah ${ }^{2}$, Muhammad Shahid ${ }^{1}$, Gowhar Ali ${ }^{1}$ and Robert D. E. Sewell ${ }^{3}$
}

\begin{abstract}
Background: Synthetic and natural antioxidants including Bacopa monnieri (L.) Pennell (Scrophulariaceae) which also possess anti-dopaminergic properties, have been proposed to be useful for emetogenic chemotherapy. In this study, synthetic [N-(2-mercaptopropionyl) glycine (MPG), vitamin C (Vit-C)] and natural [grape seed proanthocyanidin (GP), B. monnieri n-butanolic fraction (BM-ButFr)] antioxidants and their combinations were evaluated against cisplatin-induced emesis in pigeons during a $24 \mathrm{~h}$ observation period.

Methods: Emesis was induced using cisplatin (7.0 mg/kg, i.v). MPG (10, 20, 30 mg/kg), Vit-C (100, 200, 300 mg/kg), GP $(50,100,150 \mathrm{mg} / \mathrm{kg})$ and BM-ButFr $(5,10,20 \mathrm{mg} / \mathrm{kg})$ and their combinations were administered i.m., $15 \mathrm{~min}$ before cisplatin administration. The number of vomiting bouts, retching, emetic latency and \% weight loss were recorded to assess antiemetic potential. Antioxidant activity was evaluated by the DPPH free radical scavenging assay (FRSA).

Results: Significant attenuation of vomiting bouts, retching, \% weight loss along with an increase in latency was produced by all the antioxidants and their combinations compared to cisplatin alone and this is the first report of this activity of GP in pigeons. Low EC 50 values in the FRSA for MPG $(67.66 \mu \mathrm{g} / \mathrm{mL})$, Vit-C $(69.42 \mu \mathrm{g} / \mathrm{mL})$, GP $(6.498 \mu \mathrm{g} / \mathrm{mL})$ and BM-ButFr $(55.61 \mu \mathrm{g} / \mathrm{mL})$ compared to BHT standard $(98.17 \mu \mathrm{g} / \mathrm{mL})$ demonstrated their radical scavenging capacity. Correlation between the antioxidant activity and antiemetic efficacy disclosed a high degree of correlation for the tested antioxidants.
\end{abstract}

Conclusion: The selected synthetic and natural antioxidants and their combinations were able to attenuate cisplatin-induced vomiting, which correlated with their potent in vitro antioxidant activity.

Keywords: Emesis, Antiemetic, Chemotherapy, Bacopa monnieri, Grape seed proanthocyanidin, N-(2-mercaptopropionyl) glycine, Vitamin C, Free radical scavenging assay, Correlation

\section{Background}

Nausea and vomiting are the most distressing and commonly occurring side effects of chemotherapeutic agents [1]. Indeed, chemotherapy can induce both acute and delayed phases of nausea and vomiting [2]. In pigeons and piglets for instance, the acute phase lasts for $8-16 \mathrm{~h}$ while the delayed phase may endure for $48-58 \mathrm{~h}$ [3]. However, in humans, the acute and delayed phases persist for 24 and 7 days, respectively [4]. The mechanisms underlying emesis have been investigated in carnivores such as ferrets, dogs [5], and cats [6], insectivores like Suncus murinus (musk shrew) [7] and Cryptotis parva (least shrew) (Soricidae) [8,9]. Similarly, birds, notably

\footnotetext{
* Correspondence: fazal_subhan@upesh.edu.pk

${ }^{1}$ Department of Pharmacy, University of Peshawar, Peshawar 25120, Khyber

Pakhtunkhwa, Pakistan

Full list of author information is available at the end of the article
}

pigeons, also display clear-cut emetic responses to copper sulphate, glucagon, digoxin [10], theophylline [11] and amantadine [12].

Cisplatin is an effective chemotherapeutic agent indicated for the management of different malignancies including ovarian [13], head and neck [14], testicular and bladder carcinomas [15]. Its use is associated with many side effects, of which vomiting is distinctly the most distressing [16]. It decreases the plasma levels of various antioxidants [17] and also generates both oxidative and nitrosative stress $[18,19]$. The oxidative stress component plays a significant role in cisplatin-induced side effects as well as various other complications [20].

Antioxidants are effective in reducing oxidative stress evoked by cisplatin [21] and they play a pivotal role in protecting against cisplatin elicited nephrotoxicity [22], 
hepatotoxicity [23] and ototoxicity [24]. A range of antioxidants including vitamin $\mathrm{C}, \mathrm{N}$-2-mercaptopropionyl glycine (MPG, also named tiopronin), glutathione and vitamin $\mathrm{E}$ are known to be effective in cisplatin emetogenesis [25]. In this context, the synthetic antioxidant MPG, reduces cisplatin and pyrogallol provoked vomiting in Suncus murinus [26]. Proanthocyanidin (GP) an antioxidant flavonoid [27], not only possesses neuroprotective activity in humans [28] and animals [29], but also initiates a reduction of pica behavior (eating of non-food substances as a model of simulated emesis) in rats [30]. Additionally, it is effective against cisplatin-induced nephrotoxicity and hepatotoxicity [31,32], provides cardioprotection and enhances cognitive performance [33]. Similarly, Bacopa monnieri (L.) Pennell (Scrophulariaceae), a reputed nootropic plant and a rich source of bacosides has strong antioxidant [34] and neuroprotective properties [35].

Although previous studies advocate the effectiveness of antioxidants as antiemetics $[25,36]$, there is no report available in the literature showing any direct correlation between antiemetic propensity and antioxidant activity in the pigeon model for emetogenesis. The aim of this study therefore was threefold: firstly to determine any antiemetic activity of selected natural and synthetic antioxidants either alone or in combination. Secondly to evaluate their antioxidant potential and thirdly, to establish any possible correlation between their antioxidant and antiemetic activities.

\section{Methods}

\section{Animals}

Pigeons of either sex (mix breed, Department of Pharmacy, University of Peshawar, Pakistan) weighing between 200$400 \mathrm{~g}$ were used. They were acclimatized $24 \mathrm{~h}$ before the start of the experiment and were maintained at $22-26{ }^{\circ} \mathrm{C}$ on a $12 \mathrm{~h}$ light-dark cycle. Food and water were provided ad libitum. The experiments were performed in accordance with the UK Animals (Scientific Procedures) Act 1986 and were approved by the Ethical Committee of the Department of Pharmacy, University of Peshawar (Reference No. 14/EC-12/Pharm).

\section{Chemicals and standards}

Cisplatin (Korea United Pharm. Inc. Korea), analytical grade methanol (Sigma-Aldrich, Switzerland), 2,2-diphenyl-1-picrylhydrazyl (DPPH; Sigma-Aldrich, Germany), vitamin C (Vit-C; Sigma-Aldrich, Germany), butylated hydroxytoluene (BHT; Sigma-Aldrich, Germany), grape seed proanthocyanidin extracts (GP; Shaanxi Run-time Biotechnology Development Co. Ltd, Xian, China), N-(2mercaptopropionyl) glycine (MPG; Sigma-Aldrich, Germany), metoclopramide (GlaxoSmithKline).
Preparation of $\boldsymbol{n}$-butanolic fraction of $B$. monnieri extract Whole plant of B. monnieri was collected in November, 2010 from Rumalee stream near Quaid-e-Azam University, Islamabad, Pakistan. It was authenticated by Prof. Dr. Muhammad Ibrar of the Department of Botany, University of Peshawar and a specimen was deposited in the herbarium of the same Department with a voucher No 7421. The aerial parts were separated, shade dried and coarsely powdered. They were extracted with methanol in a Soxhlet apparatus and further fractionated to obtain the $n$-butanolic fraction (BM-ButFr) which is reported to be rich in bacosides [37]. Bacosides are the major active constituents of $B$. monnieri and they are considered to be responsible for its myriad pharmacological properties [38].

\section{Antiemetic activity}

\section{Preparation of drug solutions}

Cisplatin was dissolved in saline at $65-75{ }^{\circ} \mathrm{C}$ with continuous shaking and was cooled before administration. GP, MPG, Vit-C and BM-ButFr were dissolved in sterile normal saline by gentle agitation and sonicated until uniform solutions were obtained. These solutions were then immediately administered by the intramuscular route (i.m.).

\section{Induction of emesis}

The maximal (100\%) emetic dose of cisplatin $(7.0 \mathrm{mg} / \mathrm{kg})$ was used as an emetic challenge [39]. It was administered intravenously via the brachial vein and pigeon behavior was recorded for $24 \mathrm{~h}$ by video recorder. Any response with or without oral expulsion of gastric contents was considered as one bout (vomiting episode) and if occurring after a gap of $1 \mathrm{~min}$ from another bout, they were considered as separate vomiting episodes with 2 up to 80 numbers of retching (emetic behaviors) [40]. The latency to first vomit, the number of bouts, retching and \% weight loss were all recorded.

\section{Drug treatment}

Emesis was induced by administering cisplatin $(7.0 \mathrm{mg} / \mathrm{kg})$ intravenously. MPG, Vit-C, GP and BMButFr or their combination as well as metoclopramide used as the standard antiemetic agent, were administered as pretreatments $15 \mathrm{~min}$ i.m. prior to cisplatin administration. The different doses of the tested compounds were selected according to previous studies $[25,39,41,42]$. The animals were divided into the following groups:

Group I: Cisplatin control (7.0 mg/kg), $n=8$. Group II: Metoclopramide (30 mg $/ \mathrm{kg}$ ), $n=8$. Group III: MPG (10, 20, and $30 \mathrm{mg} / \mathrm{kg}), n=8$ each per dose.

Group IV: Vit-C (100, 200, and $300 \mathrm{mg} / \mathrm{kg}), n=8$ each per dose. 
Group V: GP (50, 100, and $150 \mathrm{mg} / \mathrm{kg}), n=8$ each per dose.

Group VI: BM-ButFr (5, 10, and $20 \mathrm{mg} / \mathrm{kg}), n=8$ each per dose.

Group VII: Combination of MPG (10 mg/kg) plus

Vit-C $(200 \mathrm{mg} / \mathrm{kg}), n=7$.

Group VIII: Combination of BM-ButFr $(10 \mathrm{mg} / \mathrm{kg})$ plus GP $(100 \mathrm{mg} / \mathrm{kg}), n=8$.

Group IX: Combination of GP (100 mg/kg) plus Vit-C (200 $\mathrm{mg} / \mathrm{kg}), n=8$.

The percentage reduction in the frequency of cisplatininduced vomiting bouts was calculated as:

$$
\begin{aligned}
\% \text { Reduction } & =(1-\text { mean number of bouts after treatment } \\
& \div \text { mean number of bouts of untreated control }) \\
& \times 100
\end{aligned}
$$

\section{DPPH (2,2-diphenyl-1-picrylhydrazyl) free radical scavenging activity in vitro}

The antioxidant activities of MPG, Vit-C, GP and BMButFr were evaluated by the DPPH free radical scavenging assay [43, 44]. Briefly, $2.0 \mathrm{~mL}$ of methanolic $0.1 \mathrm{mM}$ $\mathrm{DPPH}$ free radical solution was added to $1.0 \mathrm{~mL}$ of different concentrations $(1.0,10,30,50,100,200,500 \mu \mathrm{g} / \mathrm{mL})$ of GP, MPG, Vit-C, BM-ButFr or standard (BHT: butylated hydroxytoluene) in methanol. The solutions were shaken thoroughly, incubated in the dark at ambient temperature for $30 \mathrm{~min}$ and absorbance was measured at $517 \mathrm{~nm}$ using a UV/Visible spectrophotometer (Lambda 25, PerkinElmer, USA). The \% scavenging of DPPH free radicals was calculated as follows:

$\%$ of DPPH free radical scavenging activity

$$
=\left[\left(\mathrm{A}_{\mathrm{I}}-\mathrm{A}_{\mathrm{II}} / \mathrm{A}_{1}\right) \times 100\right]
$$

Where $A_{I}$ is the absorbance of the control reaction and $A_{I I}$ is the absorbance in the presence of sample.
The $\mathrm{EC}_{50}$, defined as the concentration of antioxidant causing $50 \%$ loss of DPPH activity was calculated from the graph of absorbance versus respective concentrations using non-linear regression analysis. All experiments were performed in triplicate.

\section{Statistical analysis}

Data were expressed as mean \pm S.E.M $(n=7-8)$ and analyzed by one way ANOVA followed by Tukey's multiple comparison using GraphPad Prism 5 (GraphPad Software Inc. San Diego CA, USA). Correlation analysis of antioxidant activity versus antiemetic activity of each antioxidant was carried out using the Pearson's correlation and regression program in Minitab version 17.1.0 (Minitab Inc. State College, PA 16801 USA).

\section{Results}

Antiemetic activity of $\mathrm{N}$-(2-mercaptopropionyl) glycine (MPG), vitamin C (Vit-C), grape seed proanthocyanidin (GP), and $B$. monnieri $n$-butanolic fraction (BM-ButFr) as well as MPG + Vit-C, BM-ButFr + GP and GP + Vit-C combinations As shown in Fig. 1, cisplatin generated a consistently maintained number of vomiting bouts over a $24 \mathrm{~h}$ period. It also induced retching; weight loss (\%) and a decreased latency to first vomit (Table 1). Highly significant reductions in the number of bouts were found with MPG $[F(3,28)=10.62$, $P<0.0001](10,20 \mathrm{mg} / \mathrm{kg} ; 0-8 \mathrm{~h}, P<0.001)$ (Fig. 2a), Vit-C $[F(3,28)=9.985, P=0.0001](100-300 \mathrm{mg} / \mathrm{kg} ; 0-4 \mathrm{~h}$, $P<0.001)$ (Fig. 2b), GP $[F(3,28)=50.97, P<0.0001]$ (50-150 mg/kg; $0-8 \mathrm{~h}, P<0.001$ ) (Fig. 2c) and BMButFr $[F(3,28)=48.28, P<0.0001](5-20 \mathrm{mg} / \mathrm{kg} ; 0-8 \mathrm{~h}$ and $13-16 \mathrm{~h}, P<0.001$ ) (Fig. 2d). However, the reductions were less significant $(P<0.01)$ for MPG at $30 \mathrm{mg} / \mathrm{kg}$ (0-4 h; Fig. 2a), Vit-C at $200 \mathrm{mg} / \mathrm{kg}(5-8 \mathrm{~h}$; Fig. $2 \mathrm{~b})$, GP at $150 \mathrm{mg} / \mathrm{kg}(13-24 \mathrm{~h}$; Fig. 2c) and BM-ButFr at $5-20 \mathrm{mg} / \mathrm{kg}$ (17-20 h; Fig. 2d) as compared to the cisplatin control. In the case of the combinations $[F(3,27)=$ $33.55, P<0.0001]$, highly significant inhibition $(P<0.001)$ of vomiting bouts was observed up to $4 \mathrm{~h}$ after cisplatin

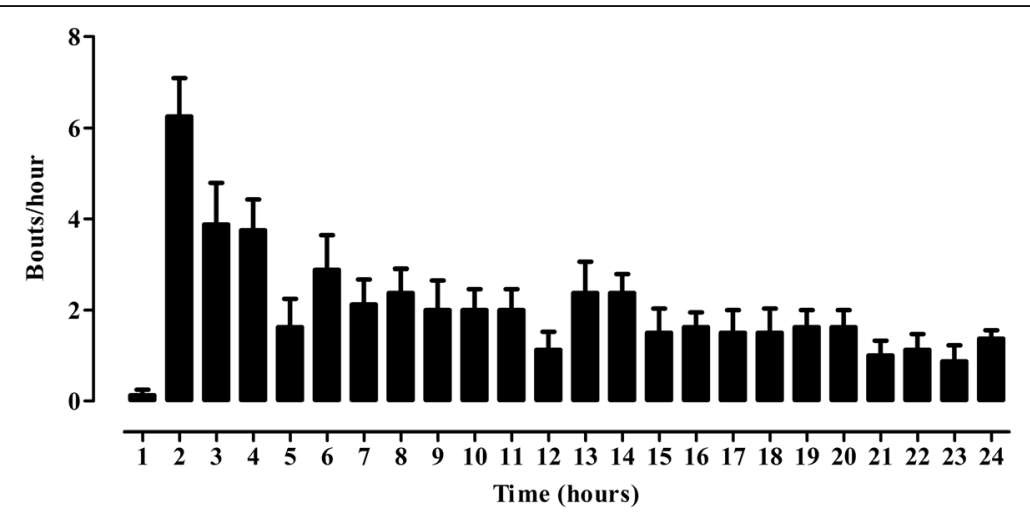

Fig. 1 Cisplatin (7.0 mg/kg i.v) induced vomiting bouts in pigeons during a $24 \mathrm{~h}$ observation period. Each bar represents mean \pm S.E.M $(n=8)$ 
Table 1 Activity of $\mathrm{N}$-(2-mercaptopropionyl) glycine (MPG), vitamin C (Vit-C), grape seed proanthocyanidin (GP), B. monnieri $n$-butanolic fraction (BM-ButFr) and their combination against cisplatin induced vomiting during a 24 h observation period

\begin{tabular}{|c|c|c|c|c|c|c|}
\hline Treatment & Dose and route & Pigeons tested/vomited & Bouts & Latency (min) & Retching & Wt. loss (\%) \\
\hline Cisplatin & $7.0 \mathrm{mg} / \mathrm{kg}$ i.v. & $8 / 8$ & $50 \pm 3.2$ & $70.0 \pm 2.30$ & $561 \pm 56.4$ & $16 \pm 1.8$ \\
\hline Metoclopramide & 30 mg/kg i.m. & $8 / 8$ & $8 \pm 0.8^{* * *}$ & $417 \pm 7.00^{* * *}$ & $134 \pm 12.5^{* * *}$ & $4 \pm 0.6^{* * *}$ \\
\hline \multirow[t]{3}{*}{ MPG } & 10 mg/kg i.m. & $8 / 6$ & $12 \pm 5.8^{* * *}$ & $410 \pm 162^{*}$ & $135+55.6^{* * *}$ & $4 \pm 1.9^{* *}$ \\
\hline & 20 mg/kg i.m. & $8 / 7$ & $21 \pm 4.9^{* *}$ & $91.0 \pm 11.5$ & $226+80.9^{*}$ & $7 \pm 1.2^{*}$ \\
\hline & 30 mg/kg i.m. & $8 / 7$ & $23 \pm 5.6^{* *}$ & $89.0 \pm 21.1$ & $329+79.2$ & $7 \pm 3.2^{*}$ \\
\hline \multirow[t]{3}{*}{ Vit-C } & 100 mg/kg i.m. & $8 / 8$ & $30 \pm 3.2^{* *}$ & $146 \pm 16.7$ & $384 \pm 23.5^{*}$ & $7 \pm 1.3^{* * *}$ \\
\hline & 200 mg/kg i.m. & $8 / 8$ & $24 \pm 4.6^{* * *}$ & $316 \pm 96.2^{*}$ & $332 \pm 52.4^{* *}$ & $5 \pm 1.1^{* * *}$ \\
\hline & 300 mg/kg i.m. & $8 / 8$ & $24 \pm 3.0^{* * *}$ & $359 \pm 73.2^{*}$ & $256 \pm 21.0^{* * *}$ & $3 \pm 0.5^{* * *}$ \\
\hline \multirow[t]{3}{*}{ GP } & 50 mg/kg i.m. & $8 / 8$ & $14 \pm 3.5^{* * *}$ & $348 \pm 42.5^{* * *}$ & $236 \pm 78.9^{* *}$ & $6 \pm 0.8^{* * *}$ \\
\hline & 100 mg/kg i.m. & $8 / 8$ & $12 \pm 2.2^{* * *}$ & $370 \pm 31.6^{* * *}$ & $184 \pm 37.0^{* * *}$ & $6 \pm 1.0^{* * *}$ \\
\hline & 150 mg/kg i.m. & $8 / 8$ & $9.0 \pm 0.9^{* * *}$ & $369 \pm 27.8^{* * *}$ & $139 \pm 27.2^{* * *}$ & $5 \pm 0.5^{* * *}$ \\
\hline \multirow[t]{3}{*}{ BM-ButFr } & $5 \mathrm{mg} / \mathrm{kg}$ i.m. & $8 / 8$ & $17 \pm 2.6^{* * *}$ & $161 \pm 25.0$ & $331 \pm 74.0$ & $7 \pm 0.5^{* *}$ \\
\hline & 10 mg/kg i.m. & $8 / 8$ & $15 \pm 2.1^{* * *}$ & $145 \pm 36.0$ & $378 \pm 58.0$ & $6 \pm 1.4^{* * *}$ \\
\hline & 20 mg/kg i.m. & $8 / 8$ & $13 \pm 1.7^{* * *}$ & $137 \pm 25.0$ & $253 \pm 45.0^{* *}$ & $5 \pm 1.7^{* * *}$ \\
\hline \multicolumn{7}{|l|}{ Combination } \\
\hline$M P G+V i t-C$ & 10 mg/kg + 200 mg/kg i.m. & $7 / 7$ & $12 \pm 4.7^{* * *}$ & $421 \pm 134^{*}$ & $201 \pm 68.2^{* * *}$ & $3 \pm 1.1^{* * *}$ \\
\hline $\mathrm{BM}-\mathrm{ButFr}+\mathrm{GP}$ & 10 mg/kg + 100 mg i.m. & $8 / 8$ & $17 \pm 0.9^{* * *}$ & $195 \pm 24.1$ & $196 \pm 16.3^{* * *}$ & $5 \pm 0.2^{* * *}$ \\
\hline$G P+V i t-C$ & 100 mg/kg + 200 mg/kg i.m. & $8 / 8$ & $9.0 \pm 3.3^{* * *}$ & $309 \pm 90.6$ & $166 \pm 61.3^{* * *}$ & $3 \pm 0.9^{* * *}$ \\
\hline
\end{tabular}

Values are expressed as mean \pm S.E.M. ${ }^{*} P<0.05,{ }^{* *} P<0.01,{ }^{* * *} P<0.001$ compared to cisplatin control (ANOVA followed by Tukey's post hoc analysis)

administration. The GP (100 mg/kg) + Vit-C (200 mg/kg) combination maintained marked significance at $5-8 \mathrm{~h}$ and during $17-20 \mathrm{~h}(P<0.001)$ whilst the other two combinations had fluctuating inhibitory activity of low statistical significance (see Fig. 2e).

A $100 \%$ reduction in the frequency of cisplatininduced vomiting bouts was observed with GP (100 and $150 \mathrm{mg} / \mathrm{kg})$ and Vit-C (300 mg/kg). Adequate protection was also produced by MPG at $10 \mathrm{mg} / \mathrm{kg}$ (72-94\%), $20 \mathrm{mg} / \mathrm{kg}(66-83 \%)$ and $30 \mathrm{mg} / \mathrm{kg}(35-60 \%)$; Vit-C at $100 \mathrm{mg} / \mathrm{kg}(28-78 \%)$ and $200 \mathrm{mg} / \mathrm{kg}(49-77 \%)$; $\mathrm{GP}$ at $50 \mathrm{mg} / \mathrm{kg}(63-97 \%)$ and BM-ButFr at $5 \mathrm{mg} / \mathrm{kg}$ (49-79\%), $10 \mathrm{mg} / \mathrm{kg}(61-78 \%)$ and $20 \mathrm{mg} / \mathrm{kg}(67-84 \%)$ during the acute phase $(0-12 \mathrm{~h})$ of cisplatin-induced vomiting. Likewise, during the delayed phase (13-24 h), the antiemetic propensity was observed as $47-60 \%$ (10 mg/kg), 0-70\% (20 mg/kg) and 3-49\% (30 mg/kg) for MPG; 0-35\% (100 mg/kg), 6-36\% (200 mg/kg) and 8$35 \%$ (300 mg/kg) for Vit-C; 41-59\% (50 mg/kg), 57-68\% $(100 \mathrm{mg} / \mathrm{kg})$ and $73-88 \%(150 \mathrm{mg} / \mathrm{kg})$ for GP and $65-74 \%$ (5 mg/kg), 57-70\% (10 mg/kg) and 61-69\% (20 mg/kg) for BM-ButFr. A highly effective percentage reduction of cisplatin-induced vomiting was afforded by the combinations of MPG (10 mg/kg) + Vit-C (200 mg/kg), BM-ButFr $(10 \mathrm{mg} / \mathrm{kg})+\mathrm{GP}(100 \mathrm{mg} / \mathrm{kg})$ and GP $(100 \mathrm{mg} / \mathrm{kg})+$ Vit-C (200 $\mathrm{mg} / \mathrm{kg}$ ) as $72-83 \%, 53-86 \%$ and $54-85 \%$ during the acute phase and $62-75 \%, 53-60 \%$ and $84-92 \%$ during the delayed phase, respectively (see Additional file 1).
Extremely significant reductions in the number of retching episodes was noted for MPG $[F(3,27)=6.639$, $P=0.0016] \quad(10 \mathrm{mg} / \mathrm{kg}, P<0.001)$, Vit-C $[F(3,28)=$ 9.658, $P=0.0002](300 \mathrm{mg} / \mathrm{kg}, P<0.001)$ and GP $[F$ $(3,28)=12.70, P<0.0001] \quad(100,150 \mathrm{mg} / \mathrm{kg}, P<0.001)$ while less significant decrements were observed with MPG at $20 \mathrm{mg} / \mathrm{kg}(P<0.05)$, Vit-C at $100 \mathrm{mg} / \mathrm{kg}(P<0.05)$ and $200 \mathrm{mg} / \mathrm{kg}(P<0.01), \mathrm{GP}$ at $50 \mathrm{mg} / \mathrm{kg}(P<0.01)$ and BM-ButFr $[F(3,28)=4.799, P=0.0080]$ at $20 \mathrm{mg} /$ $\mathrm{kg}(P<0.01)$. Moreover, the reductions in the \% weight loss were very significant with BM-ButFr $[F(3,28)=10.12$, $P=0.0001]$ at $10,20 \mathrm{mg} / \mathrm{kg}(P<0.001), \mathrm{GP}[F(3,28)=$ 18.03, $P<0.0001]$ and Vit-C $[F(3,28)=20.98, P<0.0001]$ at all tested doses $(P<0.001)$. However, the decreases were less significant for MPG $[F(3,28)=4.938, P=0.0071]$ at $10 \mathrm{mg} / \mathrm{kg}(P<0.01), 20 \mathrm{mg} / \mathrm{kg}(P<0.05)$ and $30 \mathrm{mg} / \mathrm{kg}$ $(P<0.05)$ and with BM-ButFr at $5 \mathrm{mg} / \mathrm{kg}(P<0.01)$. Furthermore, significant increases in vomiting latency were observed with GP $[F(3,28)=23.93, P<0.0001]$ at all doses $(P<0.001)$ but the increases were less significant for MPG $[F(3,28)=4.170, P=0.0146](10 \mathrm{mg} / \mathrm{kg}$, $P<0.05)$ and Vit-C $[F(3,28)=5.048, P=0.0064](200$, $300 \mathrm{mg} / \mathrm{kg}, P<0.05$ ) (Table 1 ). A tendency towards an increase in vomiting latency was observed with BMButFr $[F(3,28)=2.449, P=0.084]$ at all doses. In the positive control group, metoclopramide $(30 \mathrm{mg} / \mathrm{kg}) \mathrm{sig}$ nificantly alleviated $(P<0.001)$ cisplatin-induced vomiting bouts, retching and percentage weight loss, while it 


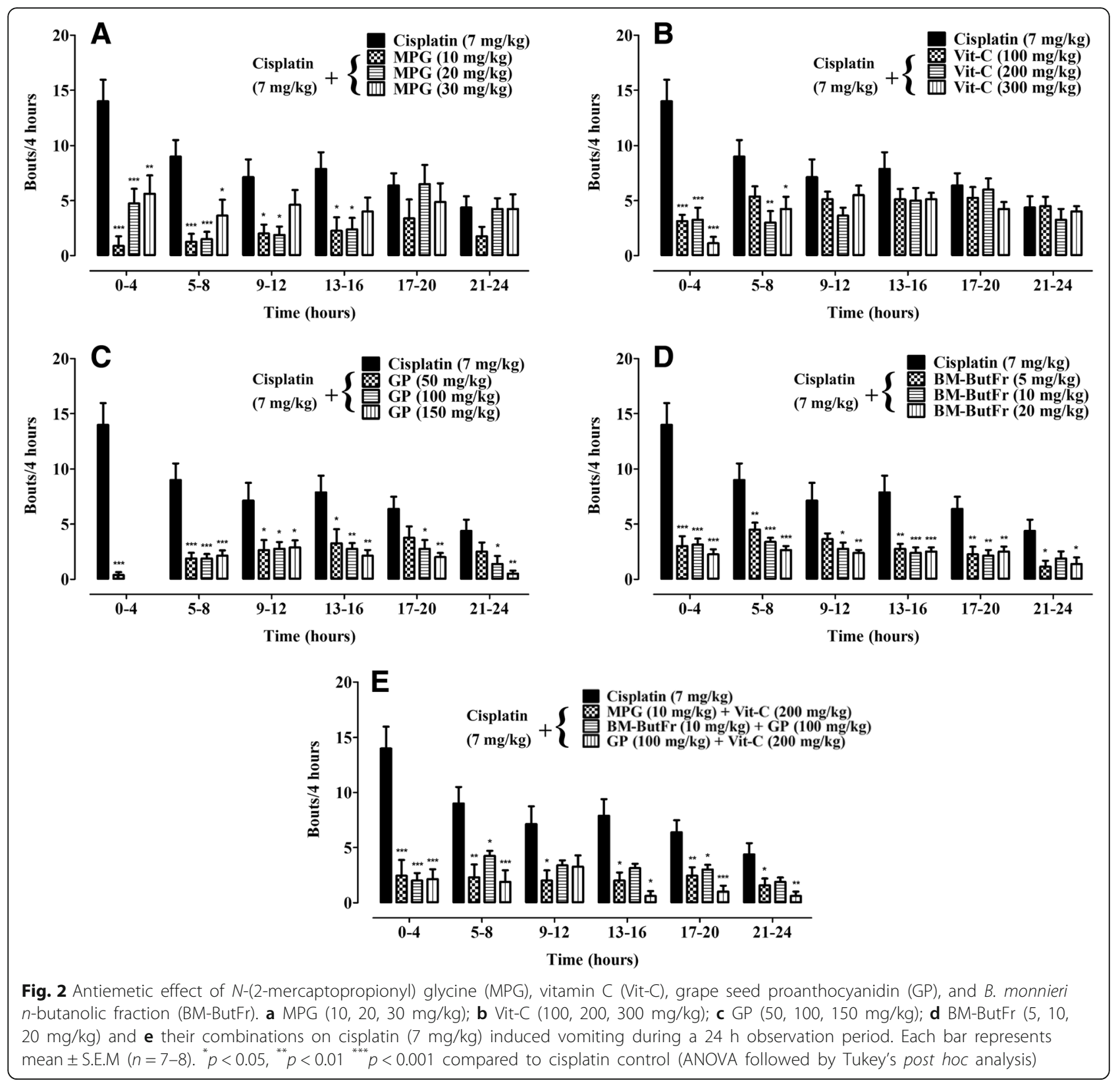

significantly increased $(P<0.001)$ the latency to vomiting during the entire observation period.

The number of vomiting bouts, retching $[F(3,27)=$ $12.54, P<0.0001]$ and $\%$ weight loss $[F(3,26)=25.72$, $P<0.0001]$ were significantly reduced $(P<0.001)$ by all the combinations of selected antioxidants when compared to the cisplatin control. In addition, the vomiting latency $[F(3,27)=3.710, P=0.0235]$ was significantly increased $(P<0.05)$ by the combination of MPG $(10 \mathrm{mg} / \mathrm{kg})+$ Vit-C $(200 \mathrm{mg} / \mathrm{kg})$ (Table 1$)$.

The global reduction in the number of emetic bouts for the selected antioxidants and their combinations decreased in the following respective rank orders: GP >
BM-ButFr $>$ MPG $>$ Vit-C and GP + Vit-C $>$ MPG + Vit$\mathrm{C}>\mathrm{BM}-\mathrm{ButFr}+\mathrm{GP}$.

In vitro antioxidant activity of $\mathrm{N}$-(2-mercaptopropionyl) glycine (MPG), vitamin C (Vit-C), grape seed proanthocyanidin (GP) and B. monnieri $n$-butanolic fraction (BM-ButFr)

The maximum inhibition of DPPH free radicals by BHT (standard) was $93.82 \%$ at $500 \mu \mathrm{g} / \mathrm{mL}$ while those of MPG, Vit-C, GP and BM-ButFr were $96.15 \%$ at $200 \mu \mathrm{g} /$ $\mathrm{mL}, 96.71 \%$ at $500 \mu \mathrm{g} / \mathrm{mL}, 92.42 \%$ at $50 \mu \mathrm{g} / \mathrm{mL}$ and $90.94 \%$ at $100 \mu \mathrm{g} / \mathrm{mL}$ respectively as shown in Table 2 . MPG, Vit-C, GP and BM-ButFr or standard (BHT) 
exhibited concentration dependent declines in spectral absorbance (Fig. 3). The $\mathrm{EC}_{50}$, antiradical power and stoichiometry of MPG, Vit-C, GP, BM-ButFr and BHT are shown in Table 2 and the antioxidant activity, established by $\mathrm{EC}_{50}$ values was decreased in the following rank order: GP > BM-ButFr $>$ MPG $>$ Vit-C $>$ BHT .

\section{Correlations between in vitro antioxidant and in vivo antiemetic activities}

The antiemetic activities in vivo were correlated with the free radical scavenging capacities of the natural and synthetic antioxidants in vitro. Figure 4 shows the high degree of Pearson correlation between the number of vomiting bouts and the \% of maximum free radical scavenging capacity of the selected antioxidants. The results manifested positive correlation coefficients for MPG $(r=0.9690)$, GP $(r=0.9926)$ and BM-ButFr $(r=0.9635)$. However, a negative correlation coefficient between the number of bouts and antioxidant activity was observed with Vit-C $(r=-0.9838)$. The coefficients of determination $\left(\mathrm{R}^{2}\right.$ : a statistical measure of how well the regression lines represent the data), disclosed an association between antioxidant activity and antiemetic assay outcome. This deduction was substantiated by the values obtained for MPG $\left(R^{2}=0.9390\right)$, Vit-C $\left(R^{2}=0.9680\right)$, GP $\left(R^{2}=0.9853\right)$ and BM-ButFr $\left(R^{2}=0.9283\right)$ (Fig. 4).

\section{Discussion}

In this study, different antioxidants of natural and synthetic origin were evaluated for their antiemetic activity against cisplatin-induced retching and vomiting in pigeons. In emetogenesis studies, different animal models including monkeys [45], pigs [46], ferrets [47], dogs [48], cats [49], house musk shrews [50] and rats [51] have been utilized for evaluating antiemetic compound activity. However, these models have some limitations in terms of cost, ease of handling, absence of a vomiting center, and inability to vomit. We have chosen the pigeon emesis model due to the fact that it expresses readily quantifiable vomiting response parameters as reported in previous studies $[3,52]$. The pigeon responds to a number of different emetic stimuli, including cardiac glycosides [53], reserpine [54], sigma receptor ligands [55], 5- $\mathrm{HT}_{3}$ receptor agonists [56] and chemotherapeutic drugs [57]. The pigeon model can also be used to assay the antiemetic activity of several classes of drugs for example $\mathrm{NK}_{1}$ receptor antagonists [58] and glucocorticoids [59].

Vomiting induced by cisplatin is biphasic with an acute phase lasting for $24 \mathrm{~h}$ and a delayed phase extending to several days [2]. In pigeons, there is no mechanistically distinct acute or delayed phase of chemotherapy-induced vomiting, although earlier studies have monitored emesis for up to $72 \mathrm{~h}$ [59]. In our investigation, we observed the animals for $24 \mathrm{~h}$ in order to comply with the ethical use of animals. The dose of cisplatin for induction of emesis varies with the animal model $[45,60,61]$. We utilized cisplatin at a dose of $7.0 \mathrm{mg} / \mathrm{kg}$ for pigeons [39] and observed a robust elevation in the number of vomiting bouts, retching and \% weight loss after $24 \mathrm{~h}$.

Cancer chemotherapy is associated with generation of reactive oxygen species [62] and oxidative stress has been implicated in the emesis caused not only by cisplatin but other chemotherapeutic drugs as well [63]. Numerous studies have shown that the active metabolite of cisplatin i.e. cis-diaqodiammineplatinum generates free radicals that release serotonin from enterochromafin cells which then stimulate $5-\mathrm{HT}_{3}$ receptors on vagal afferents and initiate the emetic reflex within the brain

Table 2 Percent of DPPH free radical scavenging activity and antioxidant strength of A-(2-mercaptopropionyl) glycine (MPG), vitamin C (Vit- C), grape seed proanthocyanidin (GP), B. monnieri n-butanolic fraction (BM-ButFr) or standard butylated hydroxytoluene $(\mathrm{BHT})$ against their respective concentrations

\begin{tabular}{|c|c|c|c|c|c|}
\hline \multirow[t]{2}{*}{ Concentration $(\mu \mathrm{g} / \mathrm{mL})$} & \multicolumn{5}{|c|}{ Percent inhibition (\%) } \\
\hline & $\mathrm{BHT}$ & MPG & Vit-C & $\mathrm{GP}$ & BM-ButFr \\
\hline 1 & $15.0 \pm 0.7$ & $20.0 \pm 2.1$ & $14.6 \pm 1.5$ & $36.4 \pm 0.8$ & $24.5 \pm 0.7$ \\
\hline 10 & $24.4 \pm 4.2$ & $20.8 \pm 1.8$ & $15.7 \pm 0.6$ & $71.6 \pm 0.7$ & $20.9 \pm 0.8$ \\
\hline 30 & $22.1 \pm 1.4$ & $19.4 \pm 1.2$ & $17.3 \pm 1.5$ & $82.6 \pm 1.2$ & $32.2 \pm 2.4$ \\
\hline 50 & $22.8 \pm 0.5$ & $24.4 \pm 1.0$ & $21.2 \pm 2.4$ & $92.4 \pm 0.1$ & $46.5 \pm 2.9$ \\
\hline 100 & $58.6 \pm 3.8$ & $94.0 \pm 0.01$ & $92.7 \pm 0.7$ & $92.4 \pm 0.2$ & $90.9 \pm 0.5$ \\
\hline 200 & $89.7 \pm 2.4$ & $96.2 \pm 0.2$ & $96.0 \pm 0.3$ & $91.6 \pm 0.3$ & $90.7 \pm 0.3$ \\
\hline 500 & $93.8 \pm 0.1$ & $96.0 \pm 0.7$ & $96.7 \pm 0.2$ & $89.6 \pm 0.2$ & $89.9 \pm 0.4$ \\
\hline \multicolumn{6}{|l|}{ Antioxidant strength } \\
\hline $\mathrm{EC}_{50}(\mu \mathrm{g} / \mathrm{mL})$ & $98.17 \pm 3.842$ & $67.66 \pm 3.095$ & $69.42 \pm 3.027$ & $6.498 \pm 0.630$ & $55.61 \pm 1.137$ \\
\hline Antiradical power & $0.0102 \pm 0.0004$ & $0.0148 \pm 0.0007$ & $0.0143 \pm 0.0005$ & $0.1567 \pm 0.0145$ & $0.0159 \pm 0.0010$ \\
\hline Stoichiometry & $196.3 \pm 7.685$ & $135.3 \pm 6.191$ & $138.8 \pm 6.053$ & $13.00 \pm 1.261$ & $111.2 \pm 2.274$ \\
\hline
\end{tabular}

Values are expressed as mean \pm S.E.M from three separate experiments 


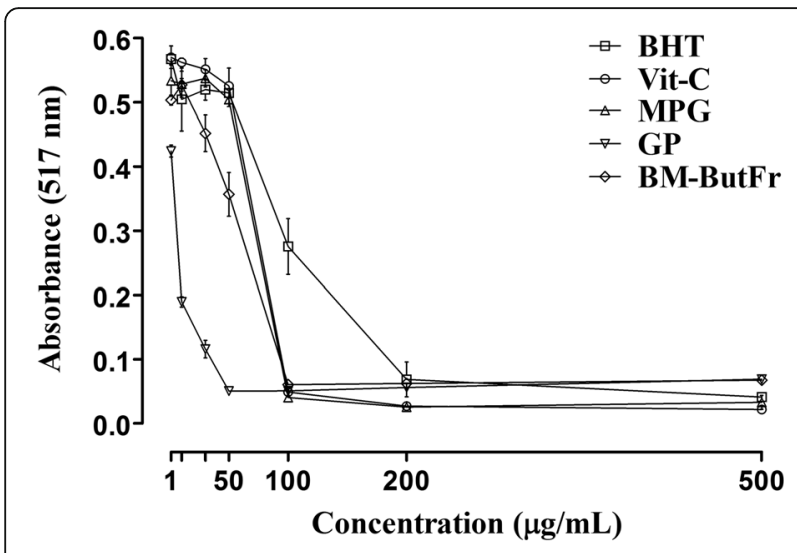

Fig. 3 DPPH free radical scavenging assay in vitro showing absorbance of $\mathrm{N}$-(2-mercaptopropionyl) glycine (MPG), vitamin C (Vit-C), grape seed proanthocyanidin (GP), B. monnieri $n$-butanolic fraction (BM-ButFr) or standard (butylated hydroxytoluene: BHT) against their respective concentrations. Data are presented as mean \pm SD of three separate experiments

stem $[64,65]$. Since the emetogenic effect of cisplatin is associated with the generation of reactive oxygen species (ROS), administration of antioxidants could detoxify ROS and thereby prevent cisplatin-induced emesis. Accordingly, antioxidants and free radical scavengers have been shown to increase the therapeutic efficacy of chemotherapy by improving tolerance and reducing their dose limiting toxicities [66]. In the present study, we have examined well established synthetic and natural antioxidants, including MPG, Vit-C, GP and BM-ButFr. Moreover, the doses chosen for these antioxidants were tolerable and benign based on their toxicity profiles [67-70].

All the selected antioxidants at the doses tested significantly decreased the number of vomiting bouts, retching and $\%$ weight loss whilst at the same time, increasing the latency to vomiting. The most intense antiemetic effect was observed with GP followed by BM-ButFr, MPG then Vit- $C$ and our study is the first to report the antiemetic activity of GP in the pigeon vomit model. GP at doses of 100 and $150 \mathrm{mg} / \mathrm{kg}$ produced a complete reversal of emesis as seen by a $100 \%$ reduction in the frequency of cisplatininduced vomiting bouts. Previously, GP at $10 \mathrm{mg} / \mathrm{kg}$ has been shown to produce a significant reduction of cisplatininduced pica behavior in rats and this is exemplified by a decreased kaolin intake [30], which is regarded as being analogous to emesis [51]. Proanthocyanidin, at $200 \mathrm{mg} / \mathrm{kg}$, ameliorated the cisplatin-induced decrease in the activities of antioxidant enzymes, GSH, total protein and albumin [31] while at $250 \mathrm{mg} / \mathrm{kg}$, it alleviated cisplatin-induced hepatotoxicity in rabbits by reducing ROS generation and strengthening endogenous antioxidant systems [32]. It is noteworthy that in rodents, investigations employing a similar dose of grape seed proanthocyanidin as that used in our study, reported beneficial effects which have been
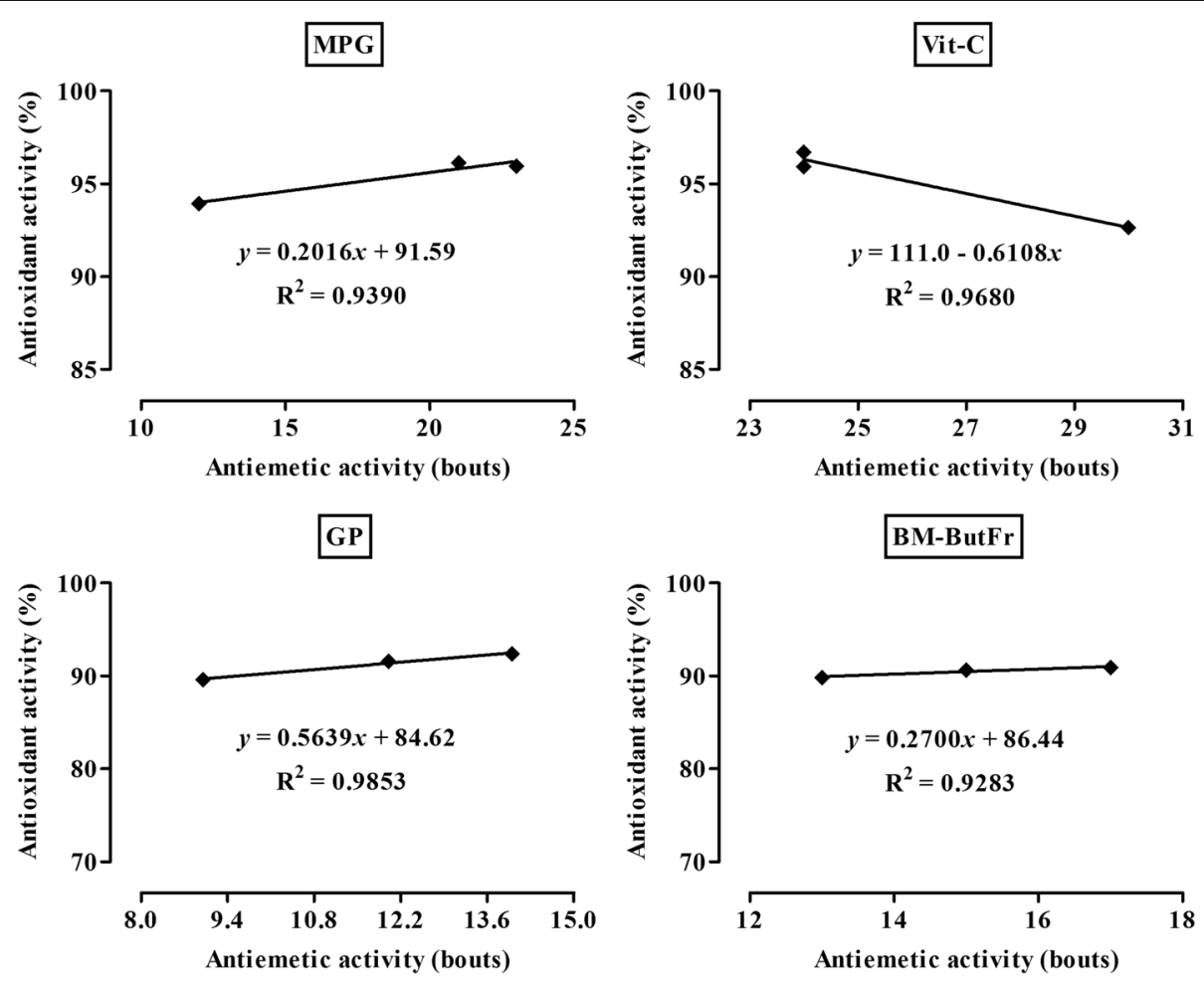

Fig. 4 Linear correlation showing the involvement of in vitro antioxidant activities of $\mathrm{N}$-(2-mercaptopropionyl) glycine (MPG), vitamin C (Vit-C), grape seed proanthocyanidin (GP) and B. monnieri $n$-butanolic fraction (BM-ButFr) in the reduction of cisplatin induced vomiting bouts in pigeons 
attributed to an ability to support the antioxidant defense system $[41,71]$.

Different plants have been screened against cisplatininduced emesis in a variety of animal models. These include Zingiber officinale Rosc. (Zingiberaceae) [48], Scutellaria baicalensis Georgi (Lamiaceae) [72] and American ginseng berry (Panax quinquefolius L.) (Araliaceae) [73]. In these studies, the antiemetic effect has been attributed to the free radical scavenging property and an antiserotonergic action of the different active constituents. In the current investigation, the $n$-butanolic fraction of B. monnieri (BM-ButFr) exhibited a dose dependant antiemetic activity and this accords with our previous report in which BM-ButFr significantly reduced cisplatin-induced emetogenesis [39]. The superior antiemetic effect of BM-ButFr compared to the synthetic antioxidants seen here, may be ascribed to the presence of bacoside-A components, which along with bacopaside I, constitute more than $96 \%$ $\mathrm{w} / \mathrm{w}$ of the total saponins present in the extract [74]. However, the antiemetic activity of $B$. monnieri may also be mediated through other mechanisms in addition to its antioxidant activity [75] because B. monnieri has both anti-dopaminergic and anti-serotonergic properties [76]. In relation to this, serotonin [77] and dopamine [78] both play an important role in the induction of vomiting at the level of the area postrema. However, serotonin has been shown to differentially mediate the early emetic phase following cisplatin treatment [77]. In line with this, our previous study showed that $B$. monnieri not only attenuated the cisplatin-induced dopamine upsurge in the area postrema and brain stem but it also diminished the intestinal serotonin concentration in the pigeons [39].

MPG is a well-known synthetic aminothiol antioxidant that has been studied widely especially for its cardioprotective properties $[78,79]$. In the present pigeon emetogenesis model, it produced a significant decline in frequencies of cisplatin-induced retching and vomiting and this is consistent with previous reports in such species as dogs [25], rats [80] and Suncus murinus [26]. It is clear that MPG has some proficiency in scavenging generated free radicals and that it exerts its beneficial effects by protecting against oxidative stress [78]. What is more, our study revealed that MPG is effective when given in lower doses since in a higher dose MPG itself causes emesis [25].

Vitamin $C$ is a versatile water soluble antioxidant that is widely used in complementary oncology [81]. In our study, it significantly attenuated cisplatin-induced emetic episodes and this is in accordance with earlier accounts describing diminished cisplatin emesis in dogs and it also inhibited kaolin consumption in cisplatin treated rats $[25,80]$. Moreover, at $300 \mathrm{mg} / \mathrm{kg}$, Vit-C produced a $100 \%$ reduction of emesis. In relation to such findings, vitamin $\mathrm{C}$ has efficacy in reducing cisplatin oxidative stress by improving antioxidant levels, repairing DNA damage and inhibiting lipid peroxidation [82].

We have screened combinations of GP + Vit-C, MPG + Vit-C and BM-ButFr + GP against cisplatin-induced retching and vomiting. Our results showed that the GP + Vit-C combination yielded an equivalent inhibition of emetic episodes to MPG + Vit-C and BM-ButFr + GP. Additionally, the combinations tended to exert comparable, or in some instances marginally improved antiemetic effects than either agent given alone. In this respect, it has been reported that combination of vitamin $\mathrm{E}$ plus vitamin $\mathrm{C}$ affords enhanced protection against emetic episodes in dogs [25]. Similarly, a combination of vitamins $\mathrm{C}$ and $\mathrm{E}$ provides superior antiemetic activity than either of the antioxidants alone in cisplatin-induced pica behavior in rats [80]. No single antiemetic is completely effective at blocking emesis in either phase, but when administered together, the antiemetic efficacy of the combination is often greater than that of each agent given individually [77].

The inherent antioxidant potential of MPG, Vit-C, GP and BM-ButFr was evaluated by the DPPH free radical scavenging assay as this method is considered as one of the standard colorimetric methods for the evaluation of antioxidant properties of natural and pure compounds [83]. The antioxidant activity of the tested compounds was quantified in terms of $\mathrm{EC}_{50}$, antiradical power and stoichiometry. Agents with a low $\mathrm{EC}_{50}$ /stoichiometry value plus high antiradical power indicated strong antioxidant activity [83, 84]. Consequently, a distinct antioxidant capability was observed for $\mathrm{GP}$ which yielded $\mathrm{EC}_{50}$, antiradical power and stoichiometry values of $6.498,0.1567$ and 13.00 respectively, and this was followed in rank order by BM-ButFr (55.61, 0.0159, 111.2), MPG (67.66, 0.0148, $135.3)$ and Vit-C $(69.42,0.0143,138.8)$. These results indicated that as compared to the standard (BHT), all the selected antioxidants possessed strong free radical scavenging capacities, as seen in previous studies [43, 85].

The rank order of antioxidant activity in vitro correlated well with in vivo antiemetic activity, GP having strong antioxidant and antiemetic proclivity among the selected agents. Several studies have reported that antioxidant properties of various plants or synthetic compounds significantly contribute to their antiemetic activities $[25,86]$. In the current study, the high degree of correlation between the antioxidant and antiemetic activities as evidenced by their coefficients of determination which implied that amelioration of cisplatin emetogenesis could at least be partially ascribed to the potent free radical scavenging capacities of GP, MPG, BM-ButFr and Vit-C. A substantial body of evidence suggests that oxidative stress is one of the triggering mechanisms in the mediation of vomiting induced by chemotherapeutic agents such as cisplatin [87, 88]. 
Cisplatin induces lipid peroxidation in the brain, liver and small intestine and releases serotonin by generating free radicals. Antioxidants scavenge the generated free radicals and protect the enterochromafin cells from oxidative injury thereby suppressing the release of serotonin in the emetogenic pathway [26]. Free radical mediated reactions are responsible for a wide range of chemotherapy-induced side effects and antioxidants are able to protect non-malignant cells and organs against some of the damaging effects of cytostatic agents [63]. Dietary supplementation with synthetic and herbal antioxidants ameliorates chemotherapy-induced oxidative stress and diminishes the development of their side effects as well as improving the overall response to therapy [89]. Our study therefore endorses the notion that free radical scavengers may be a beneficial class of prophylactic drugs against cancer chemotherapeutic drug induced emesis. However, further studies are warranted to investigate if there is direct evidence linking reactive oxygen species with oxidative/redox stress injury by assay of oxidized lipid or protein markers after cisplatin treatment. Ultimately, neurochemical analysis should be performed to further correlate the antiemetic effect of these antioxidants with behavioral parameters in this model of emesis.

\section{Conclusions}

Cisplatin treatment was associated with intense vomiting as exemplified by a significant increase in the number of emetic bouts, retching, \% weight loss and a simultaneous decrease in the emetic latency in the pigeon model. Pretreatment with MPG, Vit-C, GP, and BM-ButFr or their combinations significantly attenuated cisplatin-induced elevation of vomiting episodes. The selected agents all possessed potent free radical scavenging capability and consequent antioxidant potential as evaluated via the DPPH free radical scavenging assay. Although all agents exhibited efficacy, GP was conspicuous with respect to antiemetic and antioxidant potential. Evaluation of correlation coefficients disclosed close linear relationships between antioxidant and antiemetic propensity emphasizing the involvement of antioxidant activity in the reduction of cisplatin-induced retching and vomiting episodes for all the agents tested in the study.

\section{Additional file}

Additional file 1: Effect of synthetic and natural antioxidants on cisplatin-induced vomiting bouts. Bouts per hour graph for: N-(2-mercaptopropionyl) glycine (MPG) at $10 \mathrm{mg} / \mathrm{kg}$ (Figure S1), $20 \mathrm{mg} / \mathrm{kg}$ (Figure S2) and $30 \mathrm{mg} / \mathrm{kg}$ (Figure S3); Vitamin C (Vit-C) at $100 \mathrm{mg} / \mathrm{kg}$ (Figure S4), $200 \mathrm{mg} / \mathrm{kg}$ (Figure S5) and $300 \mathrm{mg} / \mathrm{kg}$ (Figure S6); Grape seed proanthocyanidin (GP) at $50 \mathrm{mg} / \mathrm{kg}$ (Figure S7), $100 \mathrm{mg} / \mathrm{kg}$ (Figure S8) and $150 \mathrm{mg} / \mathrm{kg}$ (Figure S9); Bacopa monnieri n-butanolic fraction (BM-ButFr) at $5 \mathrm{mg} / \mathrm{kg}$ (Figure S10), $10 \mathrm{mg} / \mathrm{kg}$ (Figure S11) and $20 \mathrm{mg} / \mathrm{kg}$ (Figure S12). (DOC $3011 \mathrm{~kb}$ )

\section{Abbreviations}

5- $\mathrm{HT}_{3}$ : Serotonin 5- $\mathrm{HT}_{3}$ receptor; $\mathrm{BHT}$ : Butylated hydroxytoluene; $\mathrm{BM}$ ButFr: Bacopa monnieri n-butanolic fraction; DPPH: 2,2-diphenyl-1picrylhydrazyl; FRSA: DPPH free radical scavenging assay; GP: Grape seed proanthocyanidin; GSH: Glutathione; MPG: N-(2-mercaptopropionyl) glycine; ROS: Reactive oxygen species; Vit-C: Vitamin C

\section{Acknowledgments}

We are thankful to the Korea United Pharm. Inc Korea for donating cisplatin active material for this study.

\section{Funding}

Not applicable.

Availability of data and materials

All data that support the findings of this study are available from the corresponding author upon reasonable request.

\section{Authors' contributions}

FS initiated the idea and guided the research group as supervisor in planning and conducting experiments throughout the research project. JA conducted the experiments and carried out calculations and statistical analysis. He also prepared the initial draft of the manuscript. IU, MS and GA assisted in the experimental work. MS also helped in the analysis and interpretation of data as well as in preparing the final version of the manuscript. RDES revised the manuscript critically for important intellectual content. All authors read and approved the final manuscript.

\section{Competing interests}

The authors declare that they have no competing interests.

\section{Consent for publication}

Not applicable.

\section{Ethics approval}

Experiments on laboratory animals were performed in accordance with the UK Animals (Scientific Procedures) Act 1986 and were approved by the Ethical Committee of the Department of Pharmacy, University of Peshawar (Reference No. 14/EC-12/Pharm).

\section{Author details}

${ }^{1}$ Department of Pharmacy, University of Peshawar, Peshawar 25120, Khyber Pakhtunkhwa, Pakistan. ${ }^{2}$ Department of Pharmacy, University of Swabi, Swabi, Pakistan. ${ }^{3}$ Cardiff School of Pharmacy and Pharmaceutical Sciences, Cardiff University, Cardiff CF103NB, UK.

Received: 6 June 2016 Accepted: 13 December 2016

Published online: 13 January 2017

\section{References}

1. Kris MG, Hesketh PJ, Somerfield MR, Feyer P, Clark-Snow R, Koeller JM, et al. American Society of Clinical Oncology guideline for antiemetics in oncology: update 2006. J Clin Oncol. 2006;24(18):2932-47.

2. Rudd J, Andrews P. Mechanisms of acute, delayed, and anticipatory emesis induced by anticancer therapies. In: Hesketh PJ (ed) Management of nausea and vomiting in cancer and cancer treatment. Sudbury: Jones and Bartlett Publishers; 2005. pp. 15-66.

3. Tanihata S, Igarashi H, Suzuki M, Uchiyama T. Cisplatin-induced early and delayed emesis in the pigeon. Br J Pharmacol. 2000;130(1):132-8.

4. Yamakuni H, Sawai-Nakayama H, Imazumi K, Maeda Y, Matsuo M, Manda T, et al. Resiniferatoxin antagonizes cisplatin-induced emesis in dogs and ferrets. Eur J Pharmacol. 2002:442(3):273-8.

5. Watson J, Gonsalves S, Fossa A, McLean S, Seeger T, Obach S, et al. The anti-emetic effects of CP-99,994 in the ferret and the dog: role of the NK1 receptor. Br J Pharmacol. 1995;115(1):84-94.

6. Andrews P, Davis C. The physiology of emesis induced by anti-cancer therapy. In: Reynolds J, Andrews PLR, Davis CJ (eds) Serotonin and the scientific basis of anti-emetic therapy. Oxford: Oxford Clinical Communications; 1995. pp. 25-49. 
7. Okada F, Saito H, Matsuki N. Blockade of motion-and cisplatin-induced emesis by a $5-\mathrm{HT}_{2}$ receptor agonist in Suncus murinus. Br J Pharmacol. 1995; 114(5):931-4.

8. Darmani NA, Crim JL, Janoyan JJ, Abad J, Ramirez J. A re-evaluation of the neurotransmitter basis of chemotherapy-induced immediate and delayed vomiting: evidence from the least shrew. Brain Res. 2009;1248:40-58.

9. Darmani NA, Wang Y, Abad J, Ray AP, Thrush GR, Ramirez J. Utilization of the least shrew as a rapid and selective screening model for the antiemetic potential and brain penetration of substance $P$ and NK 1 receptor antagonists. Brain Res. 2008;1214:58-72.

10. Uchiyama T, Kaneko A, Ito R. A simple method for the detection of emetic action using pigeons. J Med Soc Toho. 1978;25:912-4.

11. Tanihata S, Saitou Y, Saitou K, Uchiyama T. Experimental analysis of theophylline-induced emetic response in pigeons. Jpn Pharmacol Ther. 2001;29(1):19-24.

12. Saitou Y, Arakawa S-I, Saitou K-I, Tanihata S. Mechanism of amantadineinduced vomiting in the pigeon. Oyo Yakuri. 2000;59(6):111-21.

13. Muggia F. Platinum compounds 30 years after the introduction of cisplatin: implications for the treatment of ovarian cancer. Gynecol Oncol. 2009; 112(1):275-81.

14. Lorch JH, Goloubeva O, Haddad Rl, Cullen K, Sarlis N, Tishler R, et al. Induction chemotherapy with cisplatin and fluorouracil alone or in combination with docetaxel in locally advanced squamous-cell cancer of the head and neck: long-term results of the TAX 324 randomised phase 3 trial. Lancet Oncol. 2011;12(2):153-9.

15. Köberle B, Tomicic MT, Usanova S, Kaina B. Cisplatin resistance: preclinical findings and clinical implications. Biochim Biophys Acta. 2010;1806(2):172-82.

16. Fernandez-Ortega P, Caloto M, Chirveches E, Marquilles R, San Francisco J, Quesada A, et al. Chemotherapy-induced nausea and vomiting in clinical practice: Impact on patients' quality of life. Support Care Cancer. 2012 20(12):3141-8

17. Weijl N, Wipkink-Bakker A, Lentjes E, Berger H, Cleton F, Osanto S. Cisplatin combination chemotherapy induces a fall in plasma antioxidants of cancer patients. Ann Oncol. 1998;9(12):1331-7.

18. Masuda H, Tanaka T, Takahama U. Cisplatin generates superoxide anion by interaction with DNA in a cell-free system. Biochem Biophys Res Commun. 1994;203(2):1175-80

19. Yoshida M, Fukuda A, Hara M, Terada A, Kitanaka Y, Owada S. Melatonin prevents the increase in hydroxyl radical-spin trap adduct formation caused by the addition of cisplatin in vitro. Life Sci. 2003;72(15):1773-80.

20. Reuter S, Gupta SC, Chaturvedi MM, Aggarwal BB. Oxidative stress, inflammation, and cancer: how are they linked? Free Radic Biol Med. 2010; 49(11):1603-16.

21. Pace A, Savarese A, Picardo M, Maresca V, Pacetti U, Del Monte G, et al. Neuroprotective effect of vitamin E supplementation in patients treated with cisplatin chemotherapy. J Clin Oncol. 2003;21(5):927-31.

22. Sahin K, Tuzcu M, Gencoglu H, Dogukan A, Timurkan M, Sahin N, et al. Epigallocatechin-3-gallate activates $\mathrm{Nrf}_{2} / \mathrm{HO}-1$ signaling pathway in cisplatininduced nephrotoxicity in rats. Life Sci. 2010;87(7):240-5.

23. Kart A, Cigremis Y, Karaman M, Ozen H. Caffeic acid phenethyl ester (CAPE) ameliorates cisplatin-induced hepatotoxicity in rabbit. Exp Toxicol Pathol. 2010;62(1):45-52.

24. Celebi S, Gurdal MM, Ozkul MH, Yasar H, Balikci HH. The effect of intratympanic vitamin C administration on cisplatin-induced ototoxicity. Eur Arch Otorhinolaryngol. 2013;270(4):1293-7.

25. Gupta Y, Sharma S. Antiemetic activity of antioxidants against cisplatininduced emesis in dogs. Environ Toxicol Pharmacol. 1996;1(3):179-84.

26. Torii Y, Mutoh M, Saito H, Matsuki N. Involvement of free radicals in cisplatininduced emesis in Suncus murinus. Eur J Pharmacol. 1993;248(2):131-5.

27. da Silva Porto PAL, Laranjinha JAN, de Freitas VAP. Antioxidant protection of low density lipoprotein by procyanidins: structure/activity relationships. Biochem Pharmacol. 2003;66(6):947-54.

28. Bagchi D, Bagchi M, Stohs SJ, Ray SD, Sen CK, Preuss HG. Cellular protection with proanthocyanidins derived from grape seeds. Ann N Y Acad Sci. 2002; 957(1):260-70

29. Devi A, Jolitha AB, Ishii N. Grape seed proanthocyanidin extract (GSPE) and antioxidant defense in the brain of adult rats. Med Sci Monit. 2006;12(4): BR124-BR9.

30. Wang C-Z, Fishbein A, Aung HH, Mehendale SR, Chang W-T, Xie J-T, et al. Polyphenol contents in grape-seed extracts correlate with antipica effects in cisplatin-treated rats. J Altern Complement Med. 2005;11(6):1059-65.
31. Yousef MI, Saad AA, El-Shennawy LK. Protective effect of grape seed proanthocyanidin extract against oxidative stress induced by cisplatin in rats. Food Chem Toxicol. 2009;47(6):1176-83.

32. Kandemir F, Benzer E, Ozkaraca M, Ceribasi S, Yildirim NC, Ozdemir N. Protective antioxidant effects of grape seed extract in a cisplatin-induced hepatotoxicity model in rabbits. Rev Med Vet-Toulouse. 2012;163(11):539-45.

33. Devi SA, Chandrasekar BS, Manjula K, Ishii N. Grape seed proanthocyanidin lowers brain oxidative stress in adult and middle-aged rats. Exp Gerontol. 2011;46(11):958-64.

34. Kunnel Shinomol G, Raghunath N, Mukunda Srinivas Bharath M. Prophylaxis with Bacopa monnieri attenuates acrylamide induced neurotoxicity and oxidative damage via elevated antioxidant function. Cent Nerv Syst Agents Med Chem. 2013;13(1):3-12.

35. Pase MP, Kean J, Sarris J, Neale C, Scholey AB, Stough C. The cognitiveenhancing effects of Bacopa monnieri: A systematic review of randomized, controlled human clinical trials. J Altern Complement Med. 2012;18(7):647-52.

36. Sharma S, Gupta Y. Effect of antioxidants on cisplatin induced delay in gastric emptying in rats. Environ Toxicol Pharmacol. 1997;3(1):41-6.

37. Kahol AP, Singh T, Tandon S, Gupta MM, Khanuja SPS. Process for the preparation of a extract rich in bacosides from the herb Bacopa monniera. Google Patents. US patent 6833143 B1, 21 Dec 2004. http://www.google.co. in/patents/US6833143.

38. Russo A, Borrelli F. Bacopa monniera, a reputed nootropic plant: an overview. Phytomedicine. 2005;12(4):305-17.

39. Ullah I, Subhan F, Rudd JA, Rauf K, Alam J, Shahid M, et al. Attenuation of cisplatin-induced emetogenesis by standardized Bacopa monnieri extracts in the pigeon: Behavioral and neurochemical correlations. Planta Med. 2014; 80(17):1569-79.

40. Preziosi P, D'Amato M, Del Carmine R, Martire M, Pozzoli G, Navarra P. The effects of $5-\mathrm{HT}_{3}$ receptor antagonists on cisplatin-induced emesis in the pigeon. Eur J Pharmacol. 1992;221(2):343-50.

41. Bagchi D, Garg A, Krohn R, Bagchi M, Bagchi D, Balmoori J, et al. Protective effects of grape seed proanthocyanidins and selected antioxidants against TPA-induced hepatic and brain lipid peroxidation and DNA fragmentation, and peritoneal macrophage activation in mice. Gen Pharmacol-Vasc S. 1998; 30(5):771-6.

42. Ding $Y$, Dai $X$, Jiang $Y$, Zhang $Z$, Bao L, Li Y, et al. Grape seed proanthocyanidin extracts alleviate oxidative stress and ER stress in skeletal muscle of low-dose streptozotocin-and high-carbohydrate/high-fat dietinduced diabetic rats. Mol Nutr Food Res. 2013;57(2):365-9.

43. Shahid M, Subhan F. Protective effect of Bacopa monniera methanol extract against carbon tetrachloride induced hepatotoxicity and nephrotoxicity. Pharmacologyonline. 2014;2(2):18-28.

44. Shahid M, Subhan F, Ullah I, Ali G, Alam J, Shah R. Beneficial effects of Bacopa monnieri extract on opioid induced toxicity. Heliyon. 2016;2(2):e00068.

45. Fukui H, Yamamoto $M$, Sasaki S, Sato S. Involvement of $5-H_{3}$ receptors and vagal afferents in copper sulfate-and cisplatin-induced emesis in monkeys. Eur J Pharmacol. 1993;249(1):13-8.

46. Forsyth D, Yoshizawa T, Morooka N, Tuite J. Emetic and refusal activity of deoxynivalenol to swine. Appl Environ Microbiol. 1977:34(5):547-52.

47. Kamato $T$, Ito H, Nagakura $Y$, Nishida A, Yuki H, Yamano $M$, et al. Mechanisms of cisplatin-and m-chlorophenylbiguinide-induced emesis in ferrets. Eur J Pharmacol. 1993;238(2):369-76.

48. Sharma S, Kochupillai V, Gupta S, Seth S, Gupta Y. Antiemetic efficacy of ginger (Zingiber officinale) against cisplatin-induced emesis in dogs. J Ethnopharmacol. 1997;57(2):93-6.

49. Smith WL, Callaham EM, Alphin RS. The emetic activity of centrally administered cisplatin in cats and its antagonism by zacopride. J Pharm Pharmacol. 1988:40(2):142-3.

50. Kwiatkowska M, Parker LA, Burton P, Mechoulam R. A comparative analysis of the potential of cannabinoids and ondansetron to suppress cisplatininduced emesis in the Suncus murinus (house musk shrew). Psychopharmacology (Berl). 2004;174(2):254-9.

51. Takeda N, Hasegawa S, Morita M, Matsunaga T. Pica in rats is analogous to emesis: an animal model in emesis research. Pharmacol Biochem Be. 1993; 45(4):817-21.

52. Navarra P, Martire M, del Carmine R, Pozzoli G, Preziosi P. A dual effect of some $5-\mathrm{HT}_{3}$ receptor antagonists on cisplatin-induced emesis in the pigeon. Toxicol Lett. 1992;64:745-9.

53. Hanzlik P, Wood D. The mechanism of digitalis-emesis in pigeons. J Pharmacol Exp Ther. 1929;37(1):67-100. 
54. Gupta G, Dhawan B. Blockade of reserpine emesis in pigeons. Arch Int Pharmacodyn Ther. 1960;128:481-90.

55. Hudzik TJ. Sigma ligand-induced emesis in the pigeon. Pharmacol Biochem Be. 1992;41(1):215-7.

56. Wolff MC, Leander JD. Comparison of the antiemetic effects of a 5-HT 1A agonist, LY228729, and 5-HT 3 antagonists in the pigeon. Pharmacol Biochem Be. 1995;52(3):571-5.

57. Wolff MC, Leander JD. Effects of a 5-HT 1A receptor agonist on acute and delayed cyclophosphamide-induced vomiting. Eur J Pharmacol. 1997;340(2): 217-20.

58. Tanihata S, Oda S, Kakuta S, Uchiyama T. Antiemetic effect of a tachykinin NK1 receptor antagonist GR205171 on cisplatin-induced early and delayed emesis in the pigeon. Eur J Pharmacol. 2003;461(2-3):197-206.

59. Tanihata S, Oda S, Nakai S, Uchiyama T. Antiemetic effect of dexamethasone on cisplatin-induced early and delayed emesis in the pigeon. Eur J Pharmacol. 2004:484(2):311-21.

60. Gylys J, Doran K, Buyniski J. Antagonism of cisplatin induced emesis in the dog. Res Commun Chem Pathol Pharmacol. 1979;23(1):61-8.

61. Nakayama H, Yamakuni H, Higaki M, Ishikawa H, Imazumi K, Matsuo M, et al. Antiemetic activity of FK1052, a 5- $\mathrm{HT}_{3}$-and 5- $\mathrm{HT}_{4}$-receptor antagonist, in Suncus murinus and ferrets. J Pharmacol Sci. 2005:98(4):396-403.

62. Sangeetha $P$, Das $U$, Koratkar R, Suryaprabha P. Increase in free radical generation and lipid peroxidation following chemotherapy in patients with cancer. Free Radic Biol Med. 1990;8(1):15-9.

63. Weijl N, Cleton F, Osanto S. Free radicals and antioxidants in chemotherapy induced toxicity. Cancer Treat Rev. 1997;23(4):209-40.

64. Aapro M, Jordan K, Feyer P. Pathophysiology and classification of chemotherapy-induced nausea and vomiting. Prevention of nausea and vomiting in cancer patients. London: Springer Healthcare; 2013. p. 5-14.

65. Andrews PL, Horn CC. Signals for nausea and emesis: Implications for models of upper gastrointestinal diseases. Auton Neurosci. 2006;125(1):100-15.

66. Block KI, Koch AC, Mead MN, Tothy PK, Newman RA, Gyllenhaal C. Impact of antioxidant supplementation on chemotherapeutic efficacy: a systematic review of the evidence from randomized controlled trials. Cancer Treat Rev. 2007;33(5):407-18.

67. Ray S, Bagchi D, Lim PM, Bagchi M, Gross SM, Kothari SC, et al. Acute and long-term safety evaluation of a novel $1 \mathrm{H} 636$ grape seed proanthocyanidin extract. Res Commun Mol Pathol Pharmacol. 2000;109(3-4):165-97.

68. Abbas M, Subhan F, Mohani N, Rauf K, Ali G, Khan M. The involvement of opioidergic mechanisms in the activity of Bacopa monnieri extract and its toxicological studies. Afr J Pharm Pharmacol. 2011;5(8):1120-4.

69. Chiba T. Effect of sulfur-containing compounds on experimental diabetes. Vl.: Screening of hypoglycemic action of sulfur-containing compounds. Yakugaku Zasshi. 1969;89(8):1138-43.

70. Ash M, Ash I. Handbook of Preservatives. NY: Synapse Info Resources; 2004

71. Bagchi M, Milnes M, Williams C, Balmoori J, Ye X, Stohs S, et al. Acute and chronic stress-induced oxidative gastrointestinal injury in rats, and the protective ability of a novel grape seed proanthocyanidin extract. Nutr Res. 1999:19(8):1189-99.

72. Aung HH, Dey L, Mehendale S, Xie J-T, Wu JA, Yuan C-S. Scutellaria baicalensis extract decreases cisplatin-induced pica in rats. Cancer Chemother Pharmacol. 2003;52(6):453-8.

73. Mehendale S, Aung H, Wang A, Yin J-J, Wang C-Z, Xie J-T, et al. American ginseng berry extract and ginsenoside Re attenuate cisplatin-induced kaolin intake in rats. Cancer Chemother Pharmacol. 2005;56(1):63-9.

74. Deepak M, Amit A. 'Bacoside B' - the need remains for establishing identity. Fitoterapia. 2013:87(2013):7-10.

75. Bhattacharya S, Bhattacharya A, Kumar A, Ghosal S. Antioxidant activity of Bacopa monniera in rat frontal cortex, striatum and hippocampus. Phytother Res. 2000;14(3):174-9.

76. Rauf K, Subhan F, Sewell RDE. A bacoside containing Bacopa monnieri extract reduces both morphine hyperactivity plus the elevated striatal dopamine and serotonin turnover. Phytother Res. 2011;26:758-63.

77. Hesketh P, Van Belle S, Aapro M, Tattersall F, Naylor R, Hargreaves R, et al. Differential involvement of neurotransmitters through the time course of cisplatin-induced emesis as revealed by therapy with specific receptor antagonists. Eur J Cancer. 2003;39(8):1074-80.

78. M-o D, Morita T, Yamashita N, Nishida K, Yamaguchi O, Higuchi Y, et al. The antioxidant $\mathrm{N}$-2-mercaptopropionyl glycine attenuates left ventricular hypertrophy in in vivo murine pressure-overload model. J Am Coll Cardiol. 2002;39(5):907-12.
79. Tanonaka K, Iwai T, Motegi K, Takeo S. Effects of N-(2-mercaptopropionyl)glycine on mitochondrial function in ischemic-reperfused heart. Cardiovasc Res. 2003;57(2):416-25.

80. Sharma S, Gupta S, Kochupillai V, Seth S, Gupta Y. Cisplatin-induced pica behaviour in rats is prevented by antioxidants with antiemetic activity. Environ Toxicol Pharmacol. 1997:3(2):145-9.

81. Grober U. Antioxidants and other micronutrients in complementary oncology. Breast Care. 2009;4(1):13

82. Suhail N, Bilal N, Khan H, Hasan S, Sharma S, Khan F, et al. Effect of vitamins $C$ and $E$ on antioxidant status of breast-cancer patients undergoing chemotherapy. J Clin Pharm Ther. 2012;37(1):22-6.

83. Mishra K, Ojha H, Chaudhury NK. Estimation of antiradical properties of antioxidants using DPPH assay: A critical review and results. Food Chem. 2012;130(4):1036-43

84. Loo A, Jain K, Darah I. Antioxidant and radical scavenging activities of the pyroligneous acid from a mangrove plant, Rhizophora apiculata. Food Chem. 2007;104(1):300-7.

85. Chun-yang L. Measuring the antiradical efficiency of proanthocyanidin from grape seed by the DPPH - assay. J Food Sci Biotech. 2006;2:102-6.

86. Yang Y, Kinoshita K, Koyama K, Takahashi K, Tai T, Nunoura Y, et al. Novel experimental model using free radical-induced emesis for surveying antiemetic compounds from natural sources. Planta Med. 1999:65(06):574-6.

87. Johnston KD, Lu Z, Rudd JA. Looking beyond $5-\mathrm{HT}_{3}$ receptors: A review of the wider role of serotonin in the pharmacology of nausea and vomiting. Eur J Pharmacol. 2014;722:13-25

88. Miner WD, Sanger GJ, Inhibition of cisplatin-induced vomiting by selective 5-Hydroxytryptamine M-receptor antagonism. Br J Pharmacol. 1986;88(3): 497-9.

89. Conklin KA. Dietary antioxidants during cancer chemotherapy: Impact on chemotherapeutic effectiveness and development of side effects. Nutr Cancer. 2000:37(1):1-18.

\section{Submit your next manuscript to BioMed Central and we will help you at every step:}

- We accept pre-submission inquiries

- Our selector tool helps you to find the most relevant journal

- We provide round the clock customer support

- Convenient online submission

- Thorough peer review

- Inclusion in PubMed and all major indexing services

- Maximum visibility for your research

Submit your manuscript at www.biomedcentral.com/submit
) Biomed Central 